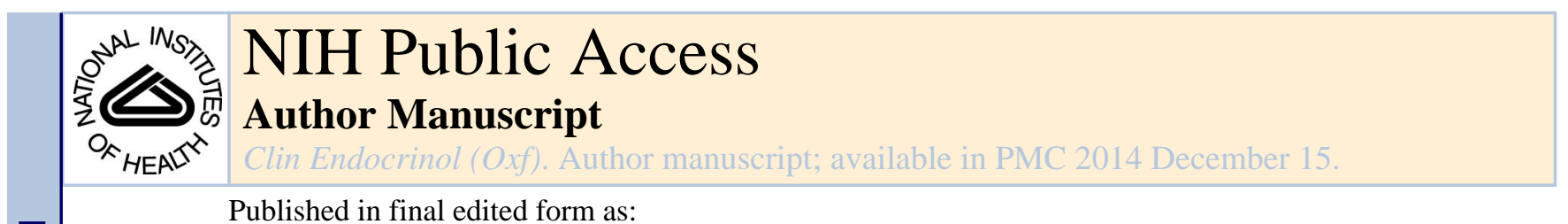

Published in final edited form as:

Clin Endocrinol (Oxf). 2012 June ; 76(6): 778-784. doi:10.1111/j.1365-2265.2011.04316.x.

\title{
Liquid chromatography-tandem mass spectrometry analysis of human adrenal vein corticosteroids before and after ACTH stimulation
}

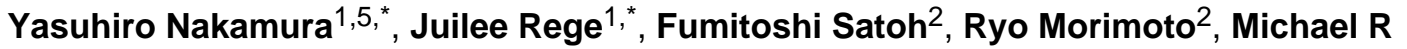 \\ Kennedy ${ }^{3}$, Clarence N Ahlem ${ }^{3}$, Seijiro Honma ${ }^{4}$, Hironobu Sasano ${ }^{5}$, and William E Rainey ${ }^{1}$ \\ ${ }^{1}$ Department of Physiology, Georgia Health Sciences University, Augusta, GA \\ 2Division of Nephrology, Endocrinology, and Vascular Medicine, Department of Medicine, Tohoku \\ University Graduate School of Medicine, Sendai, Japan \\ ${ }^{3}$ Harbor Biosciences, Inc., San Diego, CA \\ ${ }^{4}$ Aska Pharma Medical Co. Ltd., Kawasaki, Japan \\ ${ }^{5}$ Department of Pathology, Tohoku University School of Medicine, Sendai, Japan
}

\begin{abstract}
Context-Although steroid hormones produced by the adrenal gland play critical roles in human physiology, a detailed quantitative analysis of the steroid products has not been reported. The current study uses a single methodology (liquid chromatography-tandem mass spectrometry, LCMS/MS) to quantify ten corticosteroids in adrenal vein (AV) samples pre and post adrenocorticotropic hormone (ACTH) stimulation.

Design/methods-Three men and six women with a diagnosis of an adrenal aldosteroneproducing adenoma (APA) were included in the study. Serum was collected from the iliac vein (IV) and the adrenal vein (AV) contralateral to the diseased adrenal. Samples were collected, before and after administration of ACTH. LC-MS/MS was then used to quantify serum concentrations of unconjugated corticosteroids and their precursors.
\end{abstract}

Results-Prior to ACTH stimulation the four most abundant steroids in AV were cortisol (90\%), cortisone (4\%), corticosterone (3\%) and 11-deoxycortisol (0.8\%). Post ACTH administration, cortisol remained the major adrenal product (79\%), however, corticosterone became the second most abundantly produced adrenal steroid $(11 \%)$ followed by pregnenolone $(2.5 \%)$ and $17 \mathrm{a}-$ hydroxypregnenolone (2\%). ACTH significantly increased the absolute adrenal output of all ten corticosteroids measured $(P<0.05)$. The four largest post ACTH increases were pregnenolone (300-fold), progesterone (199-fold), 17a-hydroxypregnenolone (187-fold) and deoxycorticosterone (82-fold).

Correspondence and reprint requests addressed to: William E Rainey, Ph.D., Department of Physiology, Georgia Health Sciences University, 1120 15th Street, Augusta, GA 30912, Phone: 706-721-7665, FAX: 706-721-8360, wrainey@ georgiahealth.edu.

These authors contributed equally to this work 
Conclusion-Using LC-MS/MS we successfully measured 10 corticosteroids in peripheral and adrenal vein serum samples under pre and post ACTH stimulation. This study demonstrates the primary adrenal steroid products and their response to ACTH.

\section{Keywords}

adrenal vein samples; steroids; liquid chromatography-tandem mass spectrometry (LC-MS/MS)

\section{INTRODUCTION}

The human adrenal cortex is the site of the synthesis of a series of 21-carbon $\left(\mathrm{C}_{21}\right)$ steroids that include the glucocorticoids, mineralocorticoids and their precursors. ${ }^{1}$

Mineralocorticoids are produced in the zona glomerulosa (ZG) and glucocorticoids are synthesized in the zona fasciculata (ZF), respectively. ${ }^{2}$ This functional zonation results from the zone-specific expression of steroidogenic enzymes required for the synthesis of either mineralocorticoids or glucoorticoids (Figure 1) ${ }^{3-5}$ These steroids are required for life and play critical roles in the regulation of stress responses, inflammation, infection and mineral balance. Measurements of serum levels of corticosteroids, especially $\mathrm{C}_{21}$ steroids are widely utilized for the diagnoses of adrenal diseases. ${ }^{6-10}$ In addition steroid measurement in adrenal vein sampling (AVS) is currently considered the most accurate method for distinguishing between unilateral and bilateral adrenal disease, especially in patients with primary aldosteronism. ${ }^{11,12}$ Previous studies have made use of AVS to measure adrenal production of several corticosteroids in adrenal vein and peripheral blood using the methods of high performance liquid chromatography (HPLC) and/or radioimmunoassay (RIA). ${ }^{13-15}$ Most studies have focused only on the levels of aldosterone and cortisol in adrenal veins, and a detailed quantitative analysis of other products has not been reported. With growing interest in defining potential biomarkers that can be used to diagnose adrenal disease, it is important to know accurate levels of corticosteroids in peripheral and adrenal vein samples. Liquid chromatography-tandem mass spectrometry, (LC-MS/MS) is rapidly becoming the method of choice to quantify levels of steroids that are needed for the diagnosis of diseases related to adrenal and gonadal function. ${ }^{16-18}$ The current study uses a single methodology (liquid chromatography-tandem mass spectrometry, LC-MS/MS) to quantify 10 adrenal corticosteroids and their precursors in adrenal vein (AV) samples pre and post adrenocorticotropic hormone (ACTH) stimulation.

\section{MATERIALS AND METHODS}

\section{Adrenal vein sampling (AVS)}

The male $(\mathrm{n}=3)$ and female $(\mathrm{n}=6)$ patients (aged $56.4 \pm 4.4$ years) with primary aldosteronism had samples taken at Tohoku University Hospital from 2007 to 2008 via adrenal venous sampling (AVS). Informed consent was obtained from all the patients for AVS. The study was also approved by the Institutional Review Board of Tohoku University School of Medicine and Georgia Health Sciences University. The diagnosis for primary aldosteronism was established by measuring plasma aldosterone concentration/plasma renin activity (PRA) ratio which was greater than 20 at both 1 and $2 \mathrm{~h}$ after oral administration of 50mg captopril, and dexamethasone suppression tests were performed in all patients to 
exclude PA patients with cortisol-producing adenomas before AVS. ${ }^{19}$ The protocol has been previously reported in detail. ${ }^{19}$ Bilateral adrenal veins were simultaneously catheterized in all the patients. ${ }^{19}$ After baseline samples were simultaneously obtained from both adrenal veins, a second set of blood samples were collected from the same sites 15 minutes following the intravenous administration of $0.25 \mathrm{mg}$ (10 IU) of ACTH. ${ }^{19}$ Successful adrenal venous cannulation was confirmed based on the cortisol level after ACTH stimulation in the adrenal venous sample, where levels were greater than five times those found in the vena cava samples. ${ }^{19}$ The entire specimen of each resected adrenal was examined histopathologically, and all patients were diagnosed as APA. For our study, we used iliac and adrenal vein plasma from the adrenal opposite to the adrenal with an aldosteroneproducing tumor.

\section{Preparation for reagents}

Deuterium labeled corticosterone and pregnenolone were purchased from CDN Isotopes, (Pointe-Claire, Canada). Deuterium labeled cortisol was purchased from Isosciences (King of Prussia, PA, USA). 11-deoxycorticosterone, 11-deoxycortisol, 17a-hydroxyprogesterone, 17a-hydroxypregnenolone, cortisone, progesterone and 17a-ethynyl-3 $\beta, 17 \beta$-diol-5androsten-7-one were purchased from Steraloids (Newport, RI). HPLC solvents and water were HPLC analytical grade and filtered $(0.2 \mu \mathrm{m})$ before use. All other reagents were purchased from Sigma (St. Louis, MO) and were of analytical grade or better.

For aldosterone measurement, aldosterone (Aldo) and aldosterone- ${ }^{2} \mathrm{H}_{7}$ (Aldo-d7) were obtained from Sigma-Aldrich and Isosciences (King of Prussia, PA, USA), respectively. InertSep Pharma cartridge $(60 \mathrm{mg})$ and InertSep SI cartridge $(500 \mathrm{mg})$ were purchased from GL Sciences (Tokyo, Japan). Picolinic acid, 4-dimethylaminopyridine and 2-methyl-6nitrobenzoic anhydride were obtained from Tokyo Chemical Industry (Tokyo, Japan). LCMS grade acetonitrile was purchased from Wako Pure Chemicals (Osaka, Japan). All other reagents and solvents were of analytical grade.

\section{Preparation of serum samples, calibration curves and quality control (QC) samples and analysis by LC-MS/MS}

Calibration curves were prepared by serial dilution of analytical standards in water. QC samples were prepared for each steroid that was analyzed. QC samples were prepared in normal human donor plasma (one from each gender at each concentration) spiked with analyte at concentrations near the lower, mid, and upper region of the range of quantification. Calibration curves and QC samples were analyzed in parallel with AV donor samples. Steroid concentrations were calculated by Masslynx software (Waters, Beverly, MA), with the assumed concentrations for the standards calculated from the calibration curve regression parameters in comparison to theoretical values. The accuracy of QC samples was determined after first subtracting the endogenous concentration determined in native serum from the total concentration found in the QC sample.

Steroids of interest were assigned to the following categories for quantification by LCMS/MS based on the need for pre-column derivatization to achieve an appropriate lower limit quantification (LLQ) and/or chromatographic resolution: 1) no pre-column 
derivatization needed, 2) keto-steroids amenable to condensation with hydroxylamine, 3) cortisone (no pre-column derivatization), 4) aldosterone, 5) cortisol. All of the above methods used deuterium labeled internal standards where possible or $17 \alpha$-ethynyl-3 $\beta, 17 \beta$ diol-5-androsten-7-one as an internal control in the case of cortisone analysis. Calibration curves and QC samples used for each method were prepared with the corresponding precolumn derivatization method.

Regarding LC-MS/MS without pre-column derivatization (corticosterone, 11deoxycorticosterone, 11-deoxycortisol and 17a-hydroxyprogesterone) (Method 1), samples $(100 \mu \mathrm{L})$ were extracted with $4 \mathrm{~mL}$ methyl-tert-butyl-ether (MTBE). The organic phase was evaporated to dryness and reconstituted in $200 \mu \mathrm{L}$ of water/acetonitrile $(75: 25, \mathrm{v}: \mathrm{v})$. Twenty microliters of the reconstituted samples was applied to an Xterra $\mathrm{C}_{18}$ column $(2.1 \times 250 \mathrm{~mm}$, $5 \mu \mathrm{m}$, Waters, Beverly, MA), and eluted with a mobile phase gradient of $30-85 \%$ acetonitrile in water, with $0.1 \%$ formic acid. The column temperature was maintained at $40^{\circ} \mathrm{C}$. The column eluent was subjected to electrospray ionization (positive mode, $\mathrm{ES}^{+}$), and the analytes were detected with a tandem quadrupole mass spectrometer (Waters, Beverly, MA).

Regarding LC-MS/MS analysis of oxime derivatives of keto-steroids (17a-

hydroxypregnenolone, pregnenolone and progesterone) (Method 2), samples (100 $\mu \mathrm{L}$ ) were extracted with $4 \mathrm{~mL}$ MTBE, and the organic phase was evaporated to dryness. The residue was dissolved in $1 \mathrm{M}$ aqueous hydroxylamine hydrochloride, and incubated for 1 hour at $60^{\circ} \mathrm{C}$. The resulting steroid-oxime derivatives were extracted with $2.5 \mathrm{~mL}$ MTBE, dried, and reconstituted in $200 \mu \mathrm{L}$ of water/acetonitrile (75:25, v:v), and analyzed as above using a mobile phase gradient of $25-60 \%$ acetonitrile in water, with $0.1 \%$ formic acid.

Regarding LC-MS/MS analysis of cortisone (Method 3), samples (100 $\mu \mathrm{L})$ were extracted with $4 \mathrm{~mL}$ MTBE; the organic phase was evaporated to dryness under nitrogen and reconstituted in $200 \mu \mathrm{L}$ of water/acetonitrile $(75: 25, \mathrm{v}: \mathrm{v})$. Twenty microliters of the reconstituted samples was applied to a Polar RP column $(2 \times 250 \mathrm{~mm}$, Phenomenex, Torrance, CA), and eluted with a mobile phase gradient of $20-85 \%$ acetonitrile in water, with $0.1 \%$ formic acid. The column temperature was maintained at $50^{\circ} \mathrm{C}$. The column eluent was subjected to electrospray ionization (positive mode, $\mathrm{ES}^{+}$), and the analytes were detected by means of a Varian 1200L tandem quadrupole mass spectrometer (Varian, Walnut Creek, CA).

Regarding LC-MS/MS analysis of aldosterone (Method 4), an API 5000 triple stage quadrupole mass spectrometer equipped with ESI ion source (MDS-Sciex, Tronto, Canada) and a Shimadzu HPLC system (SCL-10Avp system controller, LC-20AD pump, SIL-HTc column oven, CTO-20A auto-sampler, Shimadzu, Kyoto, Japan) were employed. The column was a Cadenza CD-C18 $(250 \mathrm{~mm} \times 3 \mathrm{~mm}$ i.d. $3 \mu \mathrm{m}$, Imtakt, Kyoto, Japan $)$ and used at $45^{\circ} \mathrm{C}$. The mobile phase consisting of acetonitrile (Solvent A) and $0.1 \%$ formic acid (Solvent B) was used with a gradient elution of $\mathrm{A}: \mathrm{B}=50: 50$ to $100: 0$ at flow rate of $0.5 \mathrm{ml} /$ min. Positive electrospray ionization ion polarity was used. For quantificatoin of steroids, transition of $m / z$ 494/448 and 501/455 were selected for Aldo and Aldo-d7, respectively. Derivatizations of Aldo and Aldo-d7 were performed according to the mixed anhydride methods as previously reported. ${ }^{20}$ Deuterium labeled Aldo-d7 (100 pg) internal standard 
and acetonitrile $(0.25 \mathrm{ml})$ were added to $0.1 \mathrm{~mL}$ human serum, and the mixture was centrifuged to remove protein. The diluted supernatant was applied to an InertSep Pharma cartridge, and the steroidal fraction was eluted with $80 \%$ acetonitrile $(1 \mathrm{ml})$. After the eluate was evaporated to dryness, the residue was treated with $0.25 \mathrm{ml}$ of $35 \% \mathrm{HCl}$ in ethanol $(1: 5$, $\mathrm{vol} / \mathrm{vol}$ ) at room temperature for $30 \mathrm{~min}$. The obtained residue was reacted with mixed anhydride method for $20 \mathrm{~min}$ at $50{ }^{\circ} \mathrm{C}$. The resulting mixture was transferred to an InertSep SI cartridge and then the cartridge was washed successively with hexane $(1 \mathrm{ml})$ and hexaneethyl acetate $(7: 3$, vol/vol, $2 \mathrm{ml})$. The desired fraction was eluted with acetone-hexane (7:3, $\mathrm{vol} / \mathrm{vol}, 2 \mathrm{ml})$ and evaporated. The residue was dissolved in $70 \%$ acetonitrile $(0.1 \mathrm{ml})$ and subjected to LC-MS/MS. The accuracy and precision for Aldo in intraassay were 87-102\% and $4-11 \%$, respectively. Those in interassay were $93-101 \%$ and $7-15 \%$, respectively. The lower limit of quantitation was $10 \mathrm{pg} / \mathrm{ml}$.

Regarding LC-MS/MS analysis of cortisol (Method 5), an API 4000 triple stage quadrupole mass spectrometer (AB SCIEX) equipped with an ESI ion source, a HPLC system (Agilent 1100Series) and an autosampler (CTC Analytics HTC-PAL) were employed. The column was a Cadenza CD-C18(250 $\mathrm{mm} \times 3 \mathrm{~mm}$ I.D., $3 \mathrm{~mm}$, Imtakt $)$ and was used at $40^{\circ} \mathrm{C}$. The mobile phase consisting of $10 \mathrm{mM}$ ammonium formate (solvent $\mathrm{A}$ ) and acetonitrile (solvent $B$ ) was used with a gradient elution of $A: B=60: 40$ (0-5 min.), 60:40 to 20:80 (5-6 min.), 20:80 (6-8 min.), 0:100 (8.1-10 min.), 60:40 (10.1-13 min.). Positive electrospray ionization ion polarity was used. For quantification of cortisol, $m / z 363.2 \rightarrow 121.2$ and $367.2 \rightarrow 121.2$ were selected for F and F-d4 respectively. Deuterium labeled F-d4 (50-4000 $\mathrm{pg}$ ) internal standard and $1 \mathrm{~mL}$ acetonitrile was added to $0.1-0.2 \mathrm{~mL}$ human serum diluted to $1 \mathrm{~mL}$ with water, and the mixture was centrifuged to remove protein. The supernatant was decanted to another tube and was evaporated. The residue was dissolved with $0.25 \mathrm{~mL}$ methanol and was diluted with $1 \mathrm{~mL}$ of water. The diluted supernatant was applied to the solid-phase cartridge column(OASIS MAX : Waters). The column was washed with $1 \mathrm{~mL}$ of $10 \mathrm{mM}$ ammonium formate and $2 \mathrm{~mL}$ of $30 \%$ methanol solution. The desired steroid was eluted with $80 \%$ methanol solution and the eluted solution was applied to the cartridge column (Oasis MCX: Waters). The desired steroid was eluted completely with additional 0.5 $\mathrm{ml}$ of $80 \%$ methanol solution and the solvent was evaporated. The residue was dissolved with $0.1 \mathrm{~mL}$ of $40 \%$ acetonitrile and then subjected to LC-MS/MS.

\section{Data analysis and statistical methods}

Results are given as means \pm SEM. Statistics were calculated using two-tail paired student $t$ test with 95\% confidence intervals in SigmaPlot 8.0 (Systat Software Inc., San Jose, CA). $P<0.05$ was considered statistically significant. Each steroid was measured from the AV samples of 9 patients.

\section{RESULTS}

Prior to ACTH stimulation, the most abundant peripheral vein steroids were cortisol (139.3 $\pm 21.4 \mathrm{nmoles} / \mathrm{L})$, cortisone $(20.9 \pm 2.58 \mathrm{nmoles} / \mathrm{L})$, and corticosterone $(1.17 \pm 0.17$ nmoles/L) (Table 1), and after ACTH stimulation, the rank order remained the same: cortisol $(439.0 \pm 27.9$ nmoles/L), cortisone $(26.6 \pm 1.69$ nmoles/L), and corticosterone $(16.2 \pm 1.72$ 
nmoles/L) (Table 1). Acute ACTH stimulation (15 min) significantly increased the production of all measured steroids except for progesterone and aldosterone (Table 1). The greatest ACTH stimulated fold changes were observed for corticosterone (17-fold), 11deoxycortisol (13-fold), 17a-hydroxyprogesterone (4-fold), and cortisol (4-fold) (Table 1).

Prior to ACTH stimulation, the rank order of AV steroid abundance was cortisol (982.9 \pm $227.8 \mathrm{nmoles} / \mathrm{L})$, cortisone $(36.0 \pm 3.56 \mathrm{nmoles} / \mathrm{L})$, corticosterone $(31.4 \pm 8.14 \mathrm{nmoles} / \mathrm{L})$, 11-deoxycortisol $(9.44 \pm 3.86 \mathrm{nmoles} / \mathrm{L})$, pregnenolone $(8.37 \pm 5.25 \mathrm{nmoles} / \mathrm{L}), 17 \mathrm{a}$ hydroxyprogesterone (7.54 \pm 3.89 nmoles/L), 17a-hydroxypregnenolone $(7.12 \pm 3.93$ nmoles $/ \mathrm{L})$, aldosterone $(3.12 \pm 1.07 \mathrm{nmoles} / \mathrm{L})$, deoxycorticosterone $(1.09 \pm 0.30 \mathrm{nmoles} / \mathrm{L})$ and progesterone $(0.70 \pm 0.33 \mathrm{nmoles} / \mathrm{L})$ (Table 2$)$. The relative production of these steroids as a percent of the total mass of the ten $\mathrm{C}_{21}$ steroids that were measured was $90.1 \%$ (cortisol), 4.2\% (cortisone), 3.0\% (corticosterone) and 0.8\% (11-Deoxycortisol) (Table 3). Post ACTH administration, cortisol remained the major adrenal product (78.8\%) (Table 3). However, the relative production of the other steroids varied. Corticosterone became the second most abundantly produced adrenal steroid (11.3\%) followed by pregnenolone $(2.5 \%)$ and 17a-hydroxypregnenolone (2.1\%) (Table 3). The concentrations of the highest steroids were $17994.6 \pm 4759.8 \mathrm{nmoles} / \mathrm{L}$ for cortisol, $2550.6 \pm 673.9 \mathrm{nmoles} / \mathrm{L}$ for corticosterone, $560.0 \pm 168.5 \mathrm{nmoles} / \mathrm{L}$ for pregnenolone and $423.2 \pm 90.9 \mathrm{nmoles} / \mathrm{L}$ for $17 \mathrm{a}-$ hydroxyprogesterone (Table 2). The mean fold changes for the four most abundant steroids in $\mathrm{AV}$ were 23-fold for cortisol, 125 -fold for corticosterone, 300-fold for pregnenolone, and 137 fold for 17a-hydroxyprogesterone (Table 2). Following ACTH stimulation all steroids were significantly higher in the adrenal vein than the iliac vein (data not shown).

To visualize individual variation in adrenal steroidogenesis the predominant six steroids were plotted for each subject (Figure 2). The line graphs denote the concentrations (nmoles/L) of cortisol, corticosterone, cortisone, 11-deoxycortisol, pregnenolone and 17ahydroxypregnenolone in the adrenal vein serum of nine subjects pre and post ACTH stimulation (15 $\mathrm{min})$, with each line denoting one individual.

\section{DISCUSSION}

The adrenal cortex is a compound endocrine gland producing mineralocorticoids and glucocorticoids both of which are necessary for life. Aldosterone, the primary mineralocorticoid and cortisol the primary human glucocorticoid are produced through the metabolism of precursor steroids by five cytochrome P450 enzymes and one steroid dehydrogenase. While much is known regarding aldosterone and cortisol, less is known regarding the potential release of precursor steroids by the human adrenal gland. Herein, in addition to measuring adrenal vein levels of aldosterone and cortisol we quantified the production of the seven precursor steroids and of cortisone in human AV samples before and after ACTH stimulation. Our findings demonstrate clearly that cortisol is by mass the dominant unconjugated steroid released by the resting and ACTH stimulated adrenal gland. However, we also show that the adrenal releases a variety of other steroids that are regulated by ACTH stimulation. 
Since the 1950's numerous $\mathrm{C}_{21}$ and $\mathrm{C}_{19}$ steroids have been isolated from the extracts of human adrenal glands. ${ }^{19,21-24}$ These studies confirmed that the adrenal possesses the capability to produce a variety of steroids. The earliest studies on human adrenal venous blood steroid analysis were carried out by Romanoff et al. in 1953 and Hudson et al. in 1955, both of whom quantified the levels of cortisol and corticosterone. ${ }^{21,25}$ Taking AV samples from patients undergoing adrenalectomy, these researchers demonstrated that cortisol was present at high levels in the AV and that its concentration was several fold higher than corticosterone. In 1959, Lombardo et al. used paper chromatography to isolate and UV spectroscopy to quantify $\mathrm{C}_{21}$ steroids that included11-deoxycortisol, cortisol and corticosterone from patients with metastatic carcinoma of the breast. ${ }^{26}$ Short, in 1960 , provided further insight into this study by isolating and quantifying five $\mathrm{C}_{21}$ steroids that included cortisol, corticosterone and cortisone from patients with metastatic carcinoma of the breast. ${ }^{23}$ The values found in this study fall within the post-ACTH range found in our current study.

The next significant milestone in AVS steroid analysis was the development of steroid radioimmunoassays (RIA), which continues to be used for diagnosis of most adrenal disease. Isolation of steroids by column, paper or thin layer chromatography followed by RIA was used in several studies for quantification of steroids from AVS. In 1986, Valenta $e t$ al. used RIA to estimate the concentrations of six $\mathrm{C}_{21}$ steroids before and after ACTH stimulation. ${ }^{27}$ According to their study, cortisol (Pre ACTH, 580 nmoles/L; Post ACTH, $16700 \mathrm{nmoles} / \mathrm{L}$ ) was the most abundant of those measured followed by $17 \mathrm{a}-$

hydroxyprogesterone (Pre ACTH, 9 nmoles/L; Post ACTH, 630 nmoles/L) and progesterone (Pre ACTH, 8 nmoles/L; Post ACTH, 530 nmoles/L) ${ }^{27}$ These levels were found to be consistent with our findings. However, corticosterone, cortisone, 11deoxycortisol, pregnenolone and 17a-hydroxypregenolone were not examined in this study. ${ }^{27}$

Immunoassays have been historically and widely used for measurement of $\mathrm{C}_{21}$ steroids in routine diagnostic and research purposes. ${ }^{10,28}$ Indeed such assays have been used in numerous studies to examine peripheral serum levels of many of the $\mathrm{C}_{21}$ steroids. ${ }^{29}$ Reference values used for peripheral levels of $\mathrm{C}_{21}$ steroids appear variable between studies, laboratories and individual assays. Such assays variation may result from steroid crossreactivity, sensitivity and existence of interassay variability. ${ }^{30,31}$ LC-MS/MS is ideally suited to identify panels of steroids in complex samples such as blood, urine, and saliva. ${ }^{32,33}$ Application of this methodology to the adrenal field has the potential of defining more broadly the steroid metabolome produced by the gland as well as defining better biomarkers that will change the way we screen and diagnose adrenal disease. Herein, the current study uses LC-MS/MS to examine the levels of adrenal $\mathrm{C}_{21}$ steroids and their precursors in peripheral and adrenal vein.

The human adrenal also represents a major source of 19 carbon $\left(\mathrm{C}_{19}\right)$ steroids. ${ }^{34}$ While this study focused only on corticosteroids and their precursors, we did examine levels of the level of what is believed the predominant adrenal $\mathrm{C}_{19}$ steroid product, dehydroepiandrosterone sulfate (DHEA-S) using immunoassay. Based on this data, DHEA$S$ represents a significant product of the human adrenal with levels in the AV that surpass 
those seen for the combined mass of all $\mathrm{C}_{21}$ steroids measured in this study. It is likely that other $\mathrm{C}_{19}$ steroids are released by the human adrenal and detailed analysis of the production of these androgens and their precursors will be needed to complete the definition of the adrenal steroid metabolome.

A potential concern regarding the current study is its use of AVS from patients diagnosed with primary aldosteronism (PA). While the AVS used for our steroid analyses was from the non-diseased adrenal, it is likely that the inappropriate aldosterone production released from the adrenal with the aldosterone-producing adenoma suppressed the overall renin / angiotensin II / aldosterone system (RAAS). This in turn would lead to down-regulation of the glomerulosa in the adrenal used for AVS, which could impact the levels of mineralocorticoids measured in our study. However, the recent development of a human aldosterone synthase (CYP11B2) antibody has allowed the visualization of aldosteroneproducing cells within the ZG of the normal human adrenal. ${ }^{4}$ These immunohistochemical studies illustrate a paucity of cells expressing CYP11B2 in the normal adrenal, likely due to the high sodium diet found in most modern cultures. Thus the relative circulating levels of aldosterone and its production by the normal adrenal represent a relative small proportion of the steroid produced by the adrenal cortex.

It should also be noted that there is certainly the potential that other $\mathrm{C}_{21}$ steroids are secreted by the human adrenal. For example, we recently demonstrated that 18-oxocortisol (18oxoF), a derivative of cortisol, can be detected in the AV serum of patients with PA and could thus serve as a useful marker for the localization and lateralization of APA in patients with PA. ${ }^{35}$ However, we also noted that the levels of 18 -oxoF in the suppressed contralateral adrenal (normal adrenal) are significantly lower than the AV levels of aldosterone making it only a minor $\mathrm{C}_{21}$ steroid product.

In summary, our study indicate that LC-MS/MS profiling of ten corticosteroids and their precursors in peripheral and adrenal vein serum samples demonstrate that the major unconjugated adrenal steroids produced under basal and acute ACTH stimulation are cortisol and corticosterone. The current study represents the use of LC-MS/MS for broad based analysis of steroids in AV samples and suggests that this method will provide an excellent metrology to further define the steroid metabolites of the human adrenal gland.

\section{Acknowledgments}

This study was supported by an NIH grant to Y.N, H.S and W.E.R (DK069950). The authors also thank Dr. Mary Bassett for her editorial assistance.

\section{References}

1. Miller WL, Auchus RJ. The molecular biology, biochemistry, and physiology of human steroidogenesis and its disorders. Endocr Rev. 2011; 32:81-151. [PubMed: 21051590]

2. Neville, AM.; O’Hare, MJ. The human adrenal cortex. Springer-Verlag; New York: 1982. Functional activity of the adrenal cortex; p. 68-98.

3. Sasano H. Localization of steroidogenic enzymes in adrenal cortex and its disorders. Endocr J. 1994; 41:471-482. [PubMed: 7889106] 
4. Nishimoto K, Nakagawa K, Li D. Adrenocortical zonation in humans under normal and pathological conditions. J Clin Endocrinol Metab. 2010; 95:2296-2305. [PubMed: 20200334]

5. Nakamura Y, Gang HX, Suzuki T, et al. Adrenal changes associated with adrenarche. Rev Endocr Metab Disord Rev. 2009; 10:19-26.

6. Lin DD, Loughlin KR. Diagnosis and management of surgical adrenal diseases. Urology. 2005; 66:476-483. [PubMed: 16140061]

7. Nieman LK. Approach to the patient with an adrenal incidentaloma. J Clin Endocrinol Metab. 2010; 95:4106-4113. [PubMed: 20823463]

8. Rossi C, Calton L, Hammond G, et al. Serum steroid profiling for congenital adrenal hyperplasia using liquid chromatography-tandem mass spectrometry. Clin Chim Acta. 2010; 411:222-228. [PubMed: 19931522]

9. West CD, Tyler FH. The impact of radioimmunoassay on our understanding of human adrenal physiology. Metabolism. 1973; 22:995-1002. [PubMed: 4354165]

10. Gatti R, Antonelli G, Prearo M, et al. Cortisol assays and diagnostic laboratory procedures in human biological fluids. Clin Biochem. 2009; 42:1205-1217. [PubMed: 19414006]

11. Mulatero P, Bertello C, Verhovez A, et al. Differential diagnosis of primary aldosteronism subtypes. Curr Hypertens Rep. 2009; 11:217-223. [PubMed: 19442332]

12. Mattsson C, Young WF Jr. Primary aldosteronism: diagnostic and treatment strategies. Nat Clin Pract Nephrol. 2006; 2:198-208. [PubMed: 16932426]

13. Tortorella C, Aragona F, Nussdorfer GG. In vivo evidence that human adrenal glands possess 11 beta-hydroxysteroid dehydrogenase activity. Life Sci. 1999; 65:2823-2827. [PubMed: 10622271]

14. Granoff AB, Abraham GE. Peripheral and adrenal venous levels of steroids in a patient with virilizing adrenal adenoma. Obstet Gynecol. 1979; 53:111-115. [PubMed: 215946]

15. Curlewis JD, Axelson M, Stone GM. Identification of the major steroids in ovarian and adrenal venous plasma of the brush-tail possum (Trichosurus vulpecula) and changes in the peripheral plasma levels of oestradiol and progesterone during the reproductive cycle. J Endocrinol. 1985; 105:53-62. [PubMed: 3989423]

16. Soldin SJ, Soldin OP. Steroid hormone analysis by tandem mass spectrometry. Clin Chem. 2009; 55:1061-1066. [PubMed: 19325015]

17. Kushnir MM, Rockwood AL, Roberts WL, et al. Liquid chromatography tandem mass spectrometry for analysis of steroids in clinical laboratories. Clin Biochem. 2011; 44:77-88. [PubMed: 20627096]

18. Kulle AE, Welzel M, Holterhus PM, et al. Principles and clinical applications of liquid chromatography - tandem mass spectrometry for the determination of adrenal and gonadal steroid hormones. J Endocrinol Invest. In press.

19. Satoh F, Abe T, Tanemoto M, et al. Localization of aldosterone-producing adrenocortical adenomas: significance of adrenal venous sampling. Hypertens Res. 2007; 30:1083-1095. [PubMed: 18250558]

20. Yamashita K, Okuyama M, Nakagawa R, et al. Development of sensitive derivatization method for aldosterone in liquid chromatography-electrospray ionization tandem mass spectrometry of corticosteroids. J Chromatogr A. 2008; 1200:114-121. [PubMed: 18561939]

21. Hudson PB, Lombardo ME. Analysis of human adrenal vein blood and adrenal glands for steroidal substances. J Clin Endocrinol Metab. 1955; 15:324-330. [PubMed: 14353997]

22. Kessler MJ. Analysis of steroids from normal and tumor tissue by HPLC. Clin Chim Acta. 1982; 125:21-30. [PubMed: 7139945]

23. Short RV. The secretion of sex hormones by the adrenal gland. Biochem Soc Symp. 1960; 18:5984. [PubMed: 14446193]

24. Grant JK. Studies on the biogenesis of the adrenal steroids. Br Med Bull. 1962; 18:99-105. [PubMed: 13901157]

25. Romanoff EB, Hudson P, Pincus G. Isolation of hydrocortisone and corticosterone from human adrenal vein blood. J Clin Endocrinol Metab. 1953; 13:1546-1548. [PubMed: 13117950]

26. Lombardo ME, McMorris C, Hudson PB. The isolation of steroidal substances from human adrenal vein blood. Endocrinology. 1959; 65:417-425. 
27. Valenta LJ, Elias AN. Steroid hormones in the adrenal venous effluents in idiopathic hirsutism under basal and stimulated conditions. Horm Metab Res. 1986; 18:710-712. [PubMed: 3023220]

28. West CD, Tyler FH. The impact of radioimmunoassay on our understanding of human adrenal physiology. Metabolism. 1973; 22:995-1002. [PubMed: 4354165]

29. Munabi AK, Feuillan P, Staton RC, et al. Adrenal steroid responses to continuous intravenous adrenocorticotropin infusion compared to bolus injection in normal volunteers. J Clin Endocrinol Metab. 1986; 63:1036-1340. [PubMed: 3018023]

30. Speiser PW. Interpretation of pediatric endocrine laboratory tests: pitfalls in steroid hormone measurements and genotyping. Pediatr Endocrinol Rev. 2007; (Suppl 1):578-583. [PubMed: 18167467]

31. Andrew R. Clinical measurement of steroid metabolism. Best Pract Res Clin Endocrinol Metab. 2001; 15:1-16. [PubMed: 11469808]

32. Magnisali P, Chalioti MB, Livadara T, et al. Simultaneous quantification of $17 \mathrm{a}-\mathrm{OH}$ progesterone, 11-deoxycortisol, $\Delta 4$-androstenedione, cortisol and cortisone in newborn blood spots using liquid chromatography-tandem mass spectrometry. J Chromatogr B Analyt Technol Biomed Life Sci. 2011; 879:1565-1572.

33. Janzen N, Peter M, Sander S, et al. Newborn screening for congenital adrenal hyperplasia: additional steroid profile using liquid chromatography-tandem mass spectrometry. J Clin Endocrinol Metab. 2007; 92:2581-2589. [PubMed: 17456574]

34. Auchus RJ. The physiology and biochemistry of adrenarche. Endocr Dev. 2011; 20:20-27. [PubMed: 21164255]

35. Nakamura Y, Satoh F, Morimoto R, et al. 18-oxocortisol measurement in adrenal vein sampling as a biomarker for subclassifying primary aldosteronism. J Clin Endocrinol Metab. 2011; 96:12721278. [PubMed: 21543435] 
Cholesterol

StAR

CYP11A1

Pregnenolone

(17 a-hydroxylase)

HSD3B2 $\downarrow$

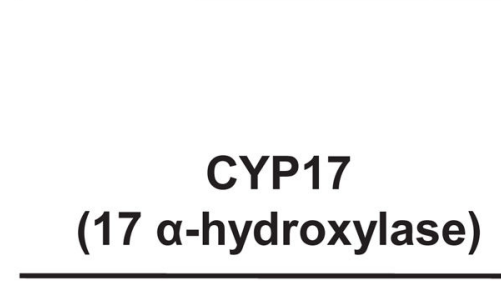

170H-Pregnenolone

CYP21

HSD3B2

(17 $\alpha$-hydroxylase)

170H-Progesterone

\section{1-Deoxycorticosterone}

CYP11B1

CYP11B2

Corticosterone

CYP11B2

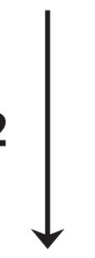

Aldosterone
CYP21

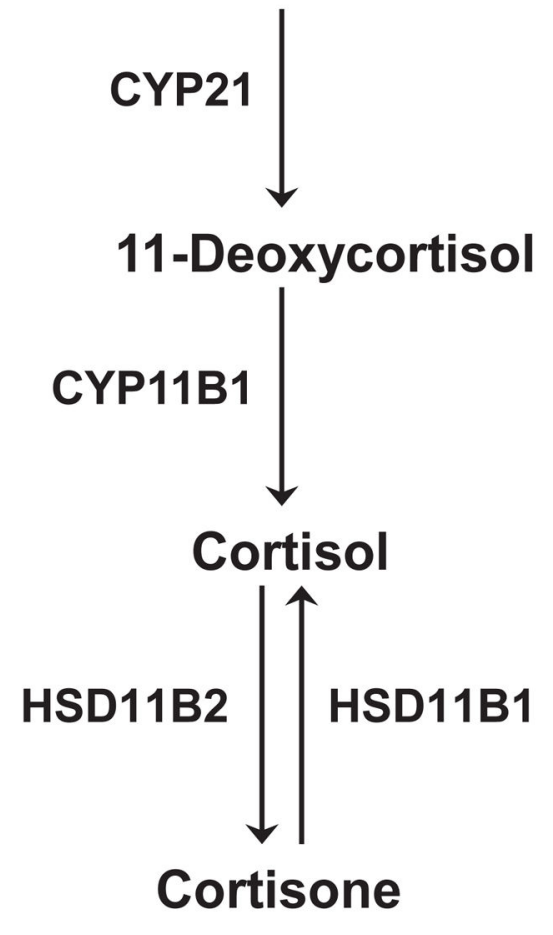

Figure 1.

Pathway for corticosteroid synthesis in the human adrenal gland. Abbreviations:

Cholesterol-side chain cleavage (CYP11A1), 33-hydroxysteroid dehydrogenase type II (HSD3B2), 17a-hydroxylase (CYP17), 21-hydroxylase (CYP21), 11ß hydroxylase (CYP11B1) and aldosterone synthase (CYP11B2), 11ß-hydroxysteroid dehydrogenase type 1 (HSD11B2), 11ß-hydroxysteroid dehydrogenase type 2 (HSD11B2). 

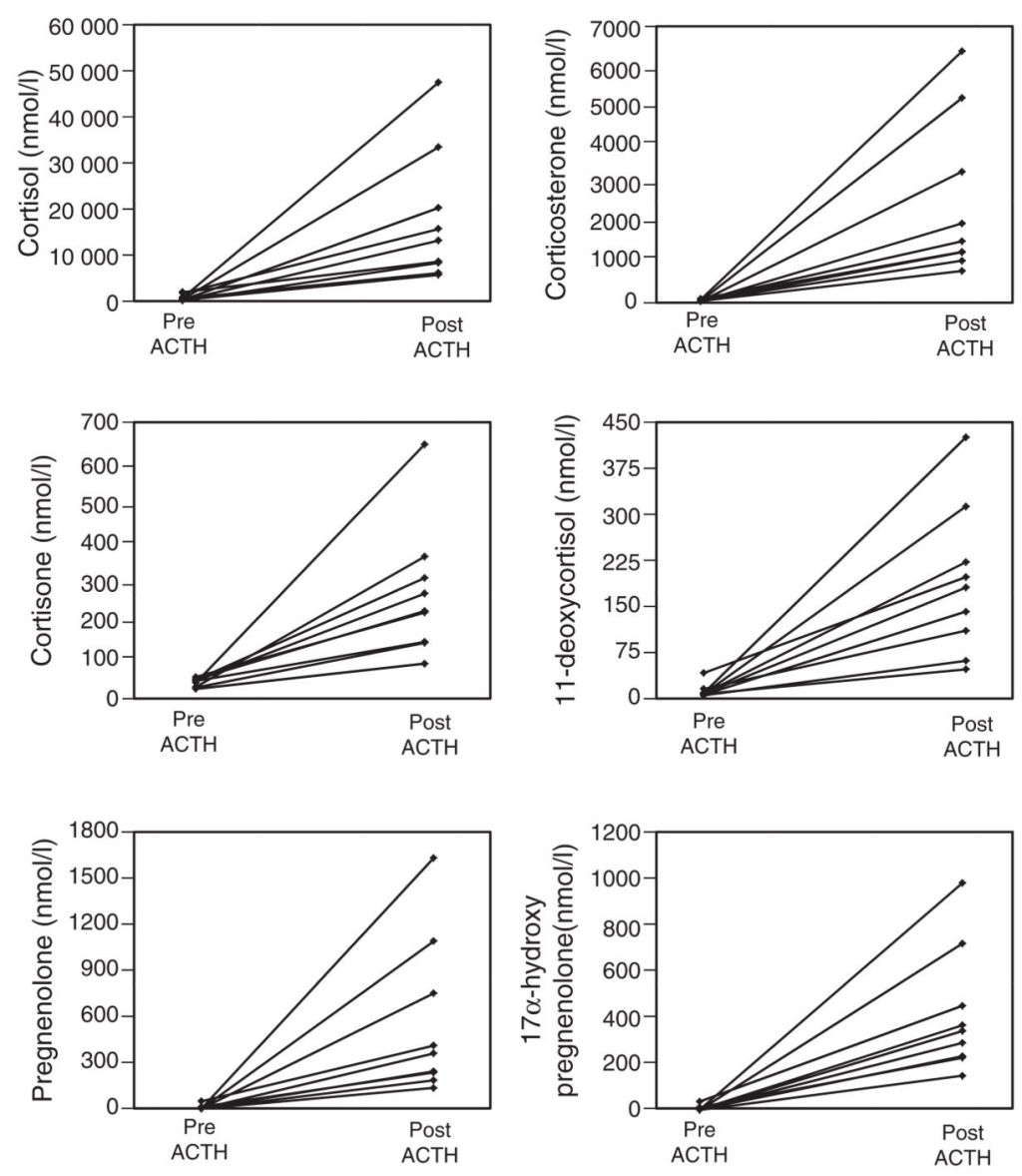

Figure 2.

Steroid concentrations (nmoles/L) were determined using LC-MS/MS for cortisol, corticosterone, cortisone, 11-deoxycortisol, pregnenolone and 17a-hydroxypregnenolone in the adrenal vein serum of nine subjects pre and post ACTH stimulation (15 min). Each line denotes the steroid increase in an individual ( $n=9,3$ men and 6 women). The line graphs denote the concentrations (nmoles/L) of cortisol, corticosterone, cortisone, 11-deoxycortisol, pregnenolone and 17a-hydroxypregnenolone in the adrenal vein serum of nine subjects pre and post ACTH stimulation (15 min), with each line denoting one individual. Individual subjects are denoted by the same line color in each panel; allowing the marked variation in production the relative steroid production varied between subjects. 


\section{TABLE 1}

Iliac vein corticosteroid levels pre- and post- ACTH infusion

\begin{tabular}{lcccc}
\hline Steroid & Pre ACTH nmoles/L & Post ACTH nmoles/L & $\boldsymbol{P}$ values & Fold Change \\
\hline Pregnenolone & $0.90 \pm 0.20$ & $1.64 \pm 0.24$ & 0.029 & $2.28 \pm 0.47$ \\
17a-Hydroxypregnenolone & $0.64 \pm 0.16$ & $1.71 \pm 0.33$ & 0.002 & $3.29 \pm 0.67$ \\
Progesterone & $0.90 \pm 0.67$ & $1.02 \pm 0.63$ & $\mathrm{NS}$ & $0.50 \pm 0.20$ \\
17a-Hydroxyprogesterone & $0.82 \pm 0.27$ & $2.08 \pm 0.47$ & $<0.001$ & $4.04 \pm 0.87$ \\
Deoxycorticosterone & $\mathrm{ND}$ & $0.41 \pm 0.09$ & 0.002 & - \\
Corticosterone & $1.17 \pm 0.17$ & $16.2 \pm 1.72$ & $<0.001$ & $17.0 \pm 3.38$ \\
Aldosterone & $0.23 \pm 0.10$ & $0.64 \pm 0.17$ & 0.003 & $3.76 \pm 0.57$ \\
11-Deoxycortisol & $0.22 \pm 0.07$ & $1.54 \pm 0.17$ & $<0.001$ & $13.0 \pm 3.04$ \\
Cortisol & $139.3 \pm 21.4$ & $439.0 \pm 27.9$ & $<0.001$ & $3.80 \pm 0.72$ \\
Cortisone & $20.9 \pm 2.58$ & $26.6 \pm 1.69$ & 0.029 & $1.34 \pm 0.15$ \\
\hline
\end{tabular}

Adrenal vein plasma samples were collected prior to ACTH infusion and 15 minutes after IV administration of 10 IU of ACTH from 9 PA patients ( 3 male, 6 female). Corticosteroids were quantified by LC-MS/MS. Values are expressed as mean \pm SEM The fold change increase following ACTH infusion is shown as the mean fold change \pm SEM. ND, not detected. 


\section{TABLE 2}

Adrenal vein corticosteroid levels pre- and post- ACTH infusion

\begin{tabular}{lllll}
\hline Steroid & Pre ACTH nmoles/L & Post ACTH nmoles/L & $P$ values & Fold Change \\
\hline Pregnenolone & $8.37 \pm 5.25$ & $560.0 \pm 168.5$ & 0.012 & $300.4 \pm 113.3$ \\
17a-Hydroxypregnenolone & $7.12 \pm 3.93$ & $414.9 \pm 88.8$ & 0.002 & $186.6 \pm 62.2$ \\
Progesterone & $0.70 \pm 0.33$ & $60.5 \pm 14.3$ & 0.003 & $199.0 \pm 68.6$ \\
17a-Hydroxyprogesterone & $7.54 \pm 3.89$ & $423.2 \pm 90.9$ & 0.002 & $137.2 \pm 36.1$ \\
Deoxycorticosterone & $1.09 \pm 0.30$ & $68.3 \pm 15.6$ & 0.002 & $82.2 \pm 19.2$ \\
Corticosterone & $31.4 \pm 8.14$ & $2550.6 \pm 673.9$ & 0.006 & $124.5 \pm 35.9$ \\
Aldosterone & $3.12 \pm 1.07$ & $24.2 \pm 5.59$ & 0.002 & $13.5 \pm 4.12$ \\
11-Deoxycortisol & $9.44 \pm 3.86$ & $187.2 \pm 40.5$ & 0.002 & $32.7 \pm 7.76$ \\
Cortisol & $982.9 \pm 227.8$ & $17994.6 \pm 4759.8$ & 0.007 & $23.4 \pm 5.55$ \\
Cortisone & $36.0 \pm 3.56$ & $262.6 \pm 55.6$ & 0.003 & $7.58 \pm 1.66$ \\
\hline
\end{tabular}

Adrenal vein plasma samples were collected prior to ACTH infusion and 15 minutes after IV administration of 10 IU of ACTH from 9 PA patients ( 3 male, 6 female). Corticosteroids were quantified by LC-MS/MS. Values are expressed as mean \pm SEM The fold change increase following $\mathrm{ACTH}$ infusion is shown as the mean fold change \pm SEM 


\section{TABLE 3}

Relative secretion of corticosteroids in AV samples

\begin{tabular}{lcc}
\hline Steroid & Pre ACTH \% of steroids measured (n=9) & Post ACTH \% of steroids measured (n=9) \\
\hline Pregnenolone & $0.56 \pm 0.19$ & $2.47 \pm 0.36$ \\
17a-Hydroxypregnenolone & $0.47 \pm 0.13$ & $2.12 \pm 0.28$ \\
Progesterone & $0.06 \pm 0.03$ & $0.30 \pm 0.05$ \\
17a-Hydroxyprogesterone & $0.51 \pm 0.13$ & $2.10 \pm 0.25$ \\
Deoxycorticosterone & $0.11 \pm 0.02$ & $0.32 \pm 0.04$ \\
Corticosterone & $2.97 \pm 0.54$ & $11.3 \pm 0.66$ \\
Aldosterone & $0.32 \pm 0.10$ & $0.13 \pm 0.03$ \\
11-Deoxycortisol & $0.77 \pm 0.12$ & $0.92 \pm 0.13$ \\
Cortisol & $90.1 \pm 0.85$ & $78.9 \pm 1.34$ \\
Cortisone & $4.23 \pm 0.64$ & $1.54 \pm 0.35$ \\
\hline
\end{tabular}

The relative production of 21-carbon corticosteroids before and $15 \mathrm{~min}$ after iv ACTH administration. Values are expressed as percentage mean \pm SEM ( $n=9,3$ men and 6 women). The percentage of each steroid was defined as the percent of the molar AV total of the 10 steroids measured. 